\title{
Effect Of Noise In The Estimation Of Magnitudes With Spatial Dependence: A Spatial Statistics Technique Based On Kriging
}

\author{
Luis Miguel Sanchez-Brea, Eusebio Bernabeu \\ Universidad Complutense de Madrid. Departamento de Optica. Facultad de Ciencias Físicas. \\ Ciudad Universitaria s.n., 28040, Madrid (Spain) \\ sanchezbrea@fis.ucm.es
}

\begin{abstract}
Kriging is a family of linear methods for the estimation of physical quantities with spatial dependence which are optimal in the squared minima sense. To perform the interpolation, kriging considers, in addition to the value and location of the observations, the spatial correlation of the quantity by means of variogram, the random fluctuations of the measured magnitude and the resolution of the measuring devices. The traditional way kriging equations are solved involves the resolution of inverse of great matrices, so that it is normally quite time consuming. Comparing the uncertainty obtained with kriging (for magnitudes with spatial dependence) with standard techniques for uncertainty estimation, we have seen that for the case of regular sampling, the uncertainty estimation can be computed as a convolution.
\end{abstract}

Keywords: Kriging, uncertainty as a convolution.

PACS: 05.40.Ca, 42.79.Pw

\section{ESTIMATION AND UNCERTAINTY OF QUANTITIES WITH SPATIAL DEPENDENCE USING KRIGING}

Kriging is a technique to estimate a quantity with spatial dependence and its uncertainty that explicitly considers the spatial correlation [1-3]. For this it performs a best linear unbiased estimation in the minimal squared sense. Kriging is widely used in geostatistics and other experimental sciences such as geology, mining, biology, medicine, etc. where few data are available, are disposed irregularly, and present strong random fluctuations. Kriging has also been applied to image processing $[4,5]$. However, in its general form, kriging equations involves inverse of matrices, which can be quite time consuming [6]. When the locations of the measuring devices are regularly disposed, Kriging equations are simplified resulting that the interpolation can be applied as a convolution [7]. With this assumption, kriging technique can be applied to image processing, including the finite size of the pixels in the design of the interpolator [8], and it has been proven to obtain better results than adaptive Wiener filter [9].

However, uncertainty estimation still needs to be solved by the conventional matrix form, even for the case of regular sampling. When the standard kriging approach is compared to the conventional statistical technique for uncertainty estimation it results 
that they do not coincide for the limit case where all the observations are placed at the same location. As a consequence, Sanchez Brea and Bernabeu [7] modified kriging equations to solve this inconvenience. It results that both equations present a similar structure.

Comparing both procedures of uncertainty estimation, in this work we propose, a convolutional method for the uncertainty estimation of magnitudes with spatial dependence. For this, we assign to each observation a function, which we have named Distributed Measurement $(D M)$ that informs us how many experimental observations should be obtained, without considering the spatial dependence, for decreasing the uncertainty as kriging (which considers the spatial dependence) does. DM function only depends on the variogram and the resolution of the measuring devices. As a result, the uncertainty of magnitudes with spatial dependence can be easily computed, without the need of performing inverse of matrices.

\section{UNCERTAINTY AS CONVOLUTION}

The traditional way that kriging performs the uncertainty estimation [1-3] does not agree, in the limit case where all observations are performed at the same location, with the standard technique for estimation the uncertainty of a magnitude $[10,11]$

$$
u^{2}=I^{2}+\frac{s^{2}}{N},
$$

being $I$ the resolution of the measuring device, $s$ the standard deviation of noise, and $N$ the number of observations. For that reason Sanchez Brea et. Bernabeu [7] have modified the equations for kriging estimation. Let us consider that we know the functional dependence of the variogram, which can be obtained using

$$
\gamma(h)=\left\langle[Z(x+h)-Z(h)]^{2}\right\rangle .
$$

Normally experimental variogram is fitted to a theoretical model of variogram, such as a Gaussian variogram. With these modifications, the proposed uncertainty estimated using kriging when $N$ observations are performed at the same location, $\mathbf{x}_{0}$, results

$$
u_{K}^{2}(\mathbf{x})=I^{2}+\frac{s^{2}}{N}+2\left[\gamma\left(\mathbf{x}-\mathbf{x}_{0}\right)-s^{2}\right] .
$$

which coincides with the definition of the uncertainty for the limit case $\mathbf{x} \rightarrow \mathbf{x}_{0}$. Comparing Eq. (3) with Eq. (1), we propose that the uncertainty of magnitudes with spatial dependence be described as

$$
u^{2}(\mathbf{x})=I^{2}+\frac{s^{2}}{N_{E Q}(\mathbf{x})} .
$$

Solving Eq. (4) it is obtained

$$
N_{E Q}(\mathbf{x})=\frac{s^{2}}{u^{2}(\mathbf{x})-I^{2}} .
$$


To determine $N_{E Q}(\mathbf{x})$ let us use the known uncertainty for just one observation. Introducing the uncertainty estimated using Eq. (3) for $N=1$ it results

$$
\left.N_{E Q}(\mathbf{x})\right|_{N=1} \rightarrow D M(\mathbf{x})=\frac{s^{2}}{2 \gamma\left(\mathbf{x}-\mathbf{x}_{0}\right)-s^{2}} .
$$

We have called this function Distributed Measurement. Since the standard deviation of a magnitude with spatial dependence can be obtained also from the variogram [12] by using $s^{2}=\lim _{h \rightarrow 0} \gamma(h)$, then to determine the uncertainty only the knowledge of the variogram and the resolution of the measuring devices is required. As a result, $D M$ function can be written as

$$
D M(\mathbf{x})=\frac{\gamma(\mathbf{0})}{2 \gamma\left(\mathbf{x}-\mathbf{x}_{0}\right)-\gamma(\mathbf{0})} .
$$

$D M$ function presents a maximum at $x=x_{0}$ and decreases when increasing the distance to the observation place, provided that the spatial correlation decreases with the distance.

We will assume that when several observations are obtained at regular locations, $N_{E Q}(\mathbf{x})$ is a linear process so it can be obtained as a sum of individual $D M(\mathbf{x})$ functions assigned to each observation.

$$
\left.N_{E Q}(\mathbf{x})\right|_{N=1}=D M(\mathbf{x}) * \amalg(\mathbf{x}),
$$

where $\amalg(\mathbf{x})=\sum_{i} \delta\left(\mathbf{x}-\mathbf{x}_{i}\right)$.
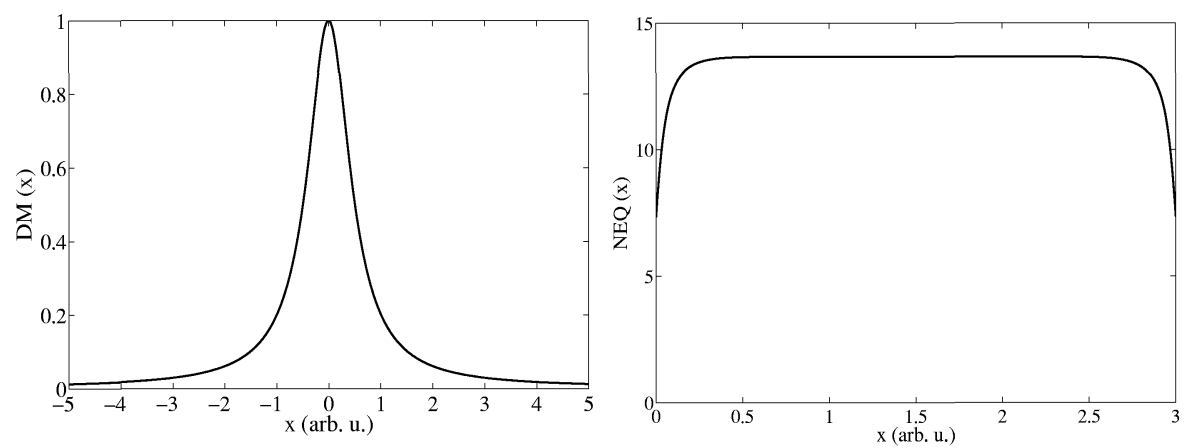

FIGURE 1. $\operatorname{DM}(\mathrm{x})$ function and $\mathrm{NEQ}(\mathrm{x})$ function for the case of a sinusoidal signal $f(x)=\sin (2 \pi x)$ with a gaussian random noise of $s=0.5$ arb.u.. The sampling frequency for determining NEQ(x) was $v=100$ arb.u. ${ }^{-1}$.

As an example of the technique, in Figure 1 we show $D M(x)$ and $N E Q(x)$ functions obtained for the case of a sinusoidal signal $f(x)=\sin (2 \pi x)$ which presents a Gaussian random noise $s=0.5 \mathrm{arb} . u$. and a resolution of $I=0.05 \mathrm{arb} . u$. In Figure 2 we show the uncertainty estimation obtained with the proposed technique, and the uncertainty obtained when the kriging estimation is compared to $f(x)$. 


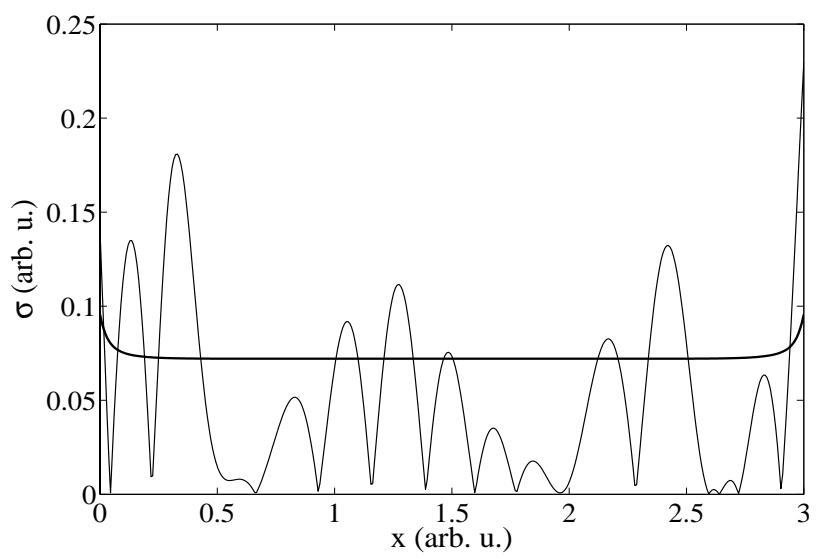

FIGURE 2. Estimation of error estimated with kriging (thin) and with Eq. (4). In this case $I=0.5$ arb.u and the standard deviation of noise (Gaussian noise) is $s=0.05$ arb.u..

\section{ACKNOWLEDGMENTS}

Sanchez-Brea is currently contracted by the Universidad Complutense de Madrid under the "Ramón y Cajal" research program.

\section{REFERENCES}

1. R. Christiensen, Linear Models for Multivariate, Time Series, and Spatial Data, Springer-Verlag, Berlin, 1985.

2. N. Cressie, Statistics for Spatial Data, John Wiley \& Sons, New York, 1991.

3. J.P. Chilès, P. Delfiner, Geostatistics: Modeling Spatial Uncertainty, John Wiley \& Sons, New York, 1999.

4. D. Mainy, J.P. Nectoux, D. Renard, Mat. Charact., 36, 327-334 (1996).

5. E. Bernabeu, I. Serroukh, L.M. Sanchez-Brea Opt. Eng., 38, 1319-1325 (1999).

6. W. H. Press, S. A. Teukolski, W. T. Vetterling, B.P. Flannery, Numerical Recipes in C, Cambridge University Press, New York, 1992.

7. L.M. Sanchez-Brea, E. Bernabeu, Appl. Opt, (in press)

8. W.Y.V. Leung, P.J. Bones, R.G. Lane, Opt. Eng., 40, 547-553 (2001).

9. T.D. Pham, M. Wagner, Int. J. Pattern Recogn., 14, 1025-1038, (2000)

10. "Guide to the Expression of the Uncertainty in Measurement", International Standardisation Organisation (ISO), Geneva, 1995.

11. P. Bevington, Data Reduction and Error Analysis for the Physical Sciences, McGraw-Hill, New York (1969).

12. L.M. Sanchez-Brea, E. Bernabeu, J. Electron. Imaging, 11, 121-126, (2002). 
Copyright of AIP Conference Proceedings is the property of American Institute of Physics. The copyright in an individual article may be maintained by the author in certain cases. Content may not be copied or emailed to multiple sites or posted to a listserv without the copyright holder's express written permission. However, users may print, download, or email articles for individual use. 